\title{
Associação do consumo regular de café da manhã e comportamentos relacionados à saúde em adolescentes
}

\author{
Association of regular consumption of breakfast and health-related \\ behavior among adolescents
}

Aline Mateus Simões (https://orcid.org/0000-0002-0816-9228) ${ }^{1}$

Christiane Opuszka Machado (https://orcid.org/0000-0002-1676-4342) ${ }^{1}$

Doroteia Aparecida Höfelmann (https://orcid.org/0000-0003-1046-3319) ${ }^{2}$

${ }^{1}$ Secretaria Municipal de Saúde, Prefeitura Municipal de Curitiba. Avenida Anita Garibaldi 6814, Barreirinha. 82220000 Curitiba PR Brasil. alinnemateussimoes@ gmail.com

${ }^{2}$ Programa de Pós-

Graduação em Alimentação

e Nutrição, Departamento de Nutrição Universidade Federal do Paraná. Curitiba PR Brasil.

\begin{abstract}
The objective of this study was to estimate the frequency of regular breakfast consumption (five or more times a week) among adolescents, and to investigate the association with demographic variables, school and job shift, health-related behaviors, eating habits and food consumption. A cross-sectional study was conducted in 30 state schools in Curitiba, State of Parana, with the participation of 1,232 adolescents. The prevalence of regular breakfast consumption was 58,6\% (95\% CI 53,9, 63.2). After adjusted analysis, higher regular breakfast consumption was observed among adolescents who: attended the evening and intermediate/full shifts; performed supervised physical activity; reported sleeping more than eight hours/night; reported having lunch and dinner with the family every day; and took 4 or more meals per day. The weekly frequency of breakfast was associated with higher consumption of milk and dairy products and the lower consumption of instant noodles and soft drinks. It is noteworthy that the habit of eating breakfast was associated with healthy eating practices and habits, reinforcing its values as a marker of other health-related behaviors. Partaking of breakfast should be encouraged among adolescents.
\end{abstract}

Key words Adolescents, Breakfast, Eating habits, Cross-sectional studies
Resumo Objetivou-se estimar a frequência do consumo regular de café da manhã (cinco ou mais vezes na semana) entre adolescentes e investigar sua associação com variáveis demográficas, turno escolar e trabalho, comportamentos relacionados à saúde, práticas alimentares e consumo de alimentos. Realizou-se estudo transversal em 30 escolas estaduais de Curitiba, Paraná, entre março de 2016 e maio de 2017, com participação de 1.232 adolescentes. A prevalência de consumo regular de café da manhã foi de 58,6\% (IC95\% 53,9; 63,2). Após análise ajustada observou-se maior consumo regular de café da manhã entre adolescentes que: frequentavam os turnos vespertino e intermediário/integral; realizavam atividade física supervisionada; relatavam dormir mais que oito horas/noite; relatavam almoçar e jantar com a família todos os dias e; realizavam quatro refeições ou mais por dia. A frequência semanal do café da manhã foi associada ao maior consumo de leite e derivados, e ao menor consumo de macarrão instantâneo e refrigerantes. Destaca-se que o hábito de realizar o desjejum esteve associado com práticas alimentares e hábitos saudáveis, reforçando seu valor enquanto marcador de outros comportamentos relacionados à saúde. Sua realização deve ser incentivada entre os adolescentes.

Palavras-chave Adolescentes, Desjejum, Hábitos alimentares, Estudos transversais 


\section{Introdução}

O café da manhã, também chamado de desjejum, é a primeira refeição do dia e, de acordo com o Guia Alimentar para a População Brasileira de 2014, é considerado uma das principais refeições diárias, assim como o almoço e o jantar ${ }^{1}$. Seu consumo regular é visto como um comportamento alimentar saudável, pois provê energia para a realização das atividades diárias após um período prolongado de jejum, e representa a refeição na qual alimentos fontes de cálcio, fibras alimentares e micronutrientes são comumente consumidos. Além disso, muitas pesquisas têm demonstrado a importância desta refeição para o desenvolvimento cognitivo, estado nutricional e qualidade da dieta ${ }^{2-4}$.

Evidências sugerem que o consumo de café da manhã pode melhorar a função cognitiva relacionada à memória, ao desempenho e à frequência escolar ${ }^{3}$. Ele está associado a diversos benefícios, o que remete a menores níveis de glicose, e menor chance de aterosclerose não coronariana ou generalizada independentemente da presença de outros fatores de risco convencionais para doença cardiovascular ${ }^{5}$. Em contrapartida, estudos têm demonstrado a associação de não tomar café da manhã com excesso de peso e obesidade em crianças ${ }^{3,6,7}$ e ao aumento do risco cardiovascular8.

A omissão de refeições é uma característica comum entre adolescentes, para quem o café da manhã é a refeição mais negligenciada. Estudos demonstram que $10 \%$ a $30 \%$ dos adolescentes nos Estados Unidos e na Europa ${ }^{3}$, e 38\% dos adolescentes brasileiros de São Paulo ${ }^{9}$ não a realizam. O perfil de não consumidores do desjejum inclui: adolescentes do sexo feminino, crianças de baixo nível socioeconômico, adolescentes mais velhos, bem como jovens negros e hispânicos ${ }^{3}$. Esta fase da vida é marcada por uma irregularidade alimentar, seja pela realização de dietas restritivas sem acompanhamento, seja pela referida falta de tempo para a realização de refeições, ou pela falta de fome nesse momento do dia ${ }^{9}$. A falta de incentivo dos pais e/ou familiares para a criação de um hábito alimentar, que inclua o consumo de café da manhã, também influencia a omissão dessa refeição entre crianças e adolescentes ${ }^{4,10}$.

Ao considerar a importância do café da manhã como uma das principais refeições do dia, a realização dessa refeição como marcador de hábitos alimentares e comportamentos saudáveis, bem como os desfechos positivos associados ao consumo regular do desjejum, o objetivo do trabalho foi estimar a frequência de consumo regular de café da manhã em adolescentes de Curitiba, Paraná, e investigar sua associação com variáveis demográficas, turno escolar e trabalho, comportamentos relacionados à saúde, práticas alimentares e consumo de alimentos.

\section{Metodologia}

Este estudo transversal, analítico, conduzido entre março de 2016 e maio de 2017 e que avaliou alunos do $6^{\circ}$ ano do Ensino Fundamental ao $3^{\circ}$ ano do Ensino Médio matriculados em instituições de ensino estadual de Curitiba, Paraná. O estudo é parte integrante do projeto "Excesso de peso e características do ambiente escolar em estudantes de Curitiba, Paraná".

Curitiba é a cidade mais populosa do Paraná, com população estimada em 2016 de 1.893.997 habitantes e Îndice de Desenvolvimento Humano (IDH) de 0,823 , considerado alto. Contudo, em 2010, 8,12\% dos domicílios particulares permanentes foram categorizados como agregados subnormais ${ }^{11}$.

A amostra calculada considerou o número de alunos matriculados do $6^{\circ}$ ano do Ensino Fundamental ao $3^{\circ}$ ano do Ensino Médio das escolas estaduais em Curitiba dos turnos diurnos $(110.238)^{12}$, prevalência desconhecida do desfecho de 50\% (para maximizar tamanho amostral), margem de erro de quatro pontos percentuais, e nível de confiança de $95 \%$, o que resultou em 597 estudantes. Como os alunos das escolas não apresentaram probabilidade igual de participar da pesquisa, o tamanho amostral obtido foi multiplicado por 2, para considerar o efeito do delineamento do estudo $(n=1.194)$. O percentual de $20 \%$ foi adicionado para compensar recusas, $\mathrm{o}$ que totalizou uma amostra de 1.433 estudantes. O cálculo de amostra foi realizado no programa OpenEpi - online com acesso livre no endereço http://www.openepi.com. Com a estimativa amostral proposta identificou-se uma razão de prevalência de 1,38 , equivalente à prevalência de $25 \%$ entre expostos e $18 \%$ não expostos, com poder de 80\% e nível de confiança de $95 \%$.

A partir do tamanho de amostra definido optou-se por realizar amostragem com aproximadamente o mesmo número de alunos por escola $(n=48)$, o que resultou no número de 30 unidades escolares. As escolas foram sorteadas da listagem total de escolas estaduais, excluídas as unidades exclusivas para educação especial ou indígena. $\mathrm{O}$ ano escolar (turma) foi sorteado em 
cada escola. Quando o número de alunos em sala era superior ao número de alunos da amostra $(n=48)$, os alunos eram sorteados, de modo alternado, iniciando com o primeiro nome da lista de chamada. Caso o número fosse inferior ao da amostra, o mesmo procedimento era realizado em duas ou mais turmas, até alcançar a quantidade de alunos necessária.

Os alunos participantes foram convidados a preencher um questionário com as variáveis: demográficas referentes a sexo (masculino, feminino) e faixa etária (10 a 13 anos, 14 a 16 anos e 17 ou mais) ${ }^{13}$; turno escolar e trabalho (matutino, vespertino, integral / intermediário para turno) e realização de trabalho remunerado (não, sim); comportamentos relacionados à saúde referente à prática de esporte com treinador (não, sim), experimentação de bebidas alcoólicas (não, sim), tabagismo (não, sim) e número de horas de sono por noite (menos de oito horas e oito horas ou mais) e; práticas alimentares pelo número de refeições diárias (até três refeições, quatro ou cinco refeições e seis ou mais refeições), hábito de realizar as refeições (almoço/jantar) em família (zero a seis dias/ todos os dias) e o local onde eram realizadas a maioria das refeições (em casa ou em outros locais).

A frequência de consumo alimentar foi estimada por meio de instrumento elaborado por Molina et al. ${ }^{14}$ e respondido pelos escolares. $\mathrm{O}$ questionário foi elaborado com base em estudos realizados no Brasil com crianças e adolescentes. Após aplicação do pré-teste foram realizadas alterações no instrumento para facilitar a compreensão e as possibilidades de análises. As modificações realizadas foram: substituição das categorias de resposta pelo número de dias na semana (zero a sete dias); adição de exemplos de alimentos nos grupos alimentares; adição de alimentos regionais junto ao grupo de tubérculos e cereais (polenta) e ao grupo de alimentos fritos (banana) e; desmembramento do item biscoito (chips/recheado) nas seguintes opções: biscoito ou bolacha doce; biscoito ou bolacha salgada e; salgadinho ou batata chips de pacote. Desta forma, os alimentos e/ou grupos investigados foram: feijão/ ervilha/lentilha; macarrão instantâneo; carnes (bovina/suína/frango); peixes/mariscos; batata, mandioca (aipim), polenta e/ou banana fritos; hortaliças cruas (alface, tomate, pepino, cebola); batata, polenta e/ou mandioca cozidos; legumes cozidos (menos batata e mandioca); recheios de pão (maionese/manteiga/margarina/requeijão); lanches prontos (hambúrguer/cachorro-quente/ pizza); leite e derivados (iogurte/queijos); frutas frescas; suco de fruta natural/polpa; refrigerante; salgados fritos (coxinha e pastel); doces/balas/ sobremesa; embutidos (presunto/salame/mortadela/linguiça), biscoito (doce ou salgado), salgadinho de pacote/batata chips, além do hábito de realizar o desjejum.

O conceito de café da manhã não foi explicitado no questionário. Ao invés disto, baseou-se na percepção do indivíduo sobre esta refeição ao responder à pergunta: "Em quantos dias da semana você toma o café da manhã, incluindo os fins de semana?", com possíveis respostas de zero a sete dias. Para a análise de dados foi considerado como desfecho o consumo regular de café da manhã (cinco vezes ou mais), enquanto o consumo até quatro vezes na semana foi considerado como não regular.

As análises descritivas foram realizadas por meio do cálculo das frequências absolutas (n), relativas (percentuais) e respectivo intervalo de confiança de 95\% (IC95\%). As associações entre o desfecho e variáveis de exposição foram estimadas por meio do teste de Wald resultante da Regressão de Poisson com cálculo das Razões de Prevalência (RP) e respectivos IC95\%. A ordem de entrada das variáveis na análise ajustada seguiu modelo hierárquico de análise: 1) demográficas; 2) turno escolar e trabalho; 3) comportamentos relacionados à saúde; 4) práticas alimentares e 5) consumo de alimentos. O critério para introdução e permanência das variáveis no modelo ajustado foi valor de $\mathrm{p}<0,25$. Desta forma, variáveis que na análise ajustada apresentaram valores superiores a 0,25 foram excluídas das análises. As diferenças foram consideradas significativas quando valor de $\mathrm{p}<0,05$. As análises foram realizadas no programa Stata 12 e foram corrigidas para efeito do delineamento e pesos amostrais (probabilidade inversa de participar em cada nível - as unidades primária e secundária de amostra foram as escolas e as turmas, respectivamente).

$\mathrm{O}$ projeto de pesquisa foi submetido à aprovação pelo Comitê de Ética em Pesquisa da Universidade Federal do Paraná. As diretrizes da Resolução do Conselho Nacional de Saúde $466 / 2012$ foram seguidas em todas as etapas da pesquisa. Os adolescentes com idade entre 12 e 18 anos receberam o Termo de Assentimento Livre e Esclarecido (TALE) na coleta de dados. O Termo de Consentimento Livre Esclarecido (TCLE) foi solicitado aos pais e/ou responsáveis. 


\section{Resultado}

Dos 1.623 alunos selecionados, 1.232 participaram da pesquisa (proporção de resposta 75,9\%). A distribuição entre os sexos foi similar $(51,0 \%$ meninos), predominantemente na faixa etária até 16 anos (90,9\%). Mais de metade dos alunos informou realizar almoço $(58,4 \%)$ ou jantar $(61,9 \%)$ com a família diariamente. A prevalência de consumo regular do café da manhã foi de $58,6 \%$ (IC95\% 53,9; 63,2), conforme demonstrado na Tabela 1. Destaca-se que $48,0 \%$ dos alunos referiram consumo diário, enquanto $17,7 \%$ não realizavam esta refeição em nenhum dia da semana.

Na Tabela 2 são apresentadas as prevalências de consumo regular do café da manhã de acordo com variáveis de estudo. Verificou-se menor

Tabela 1. Distribuição das características de adolescentes das escolas estaduais de Curitiba, Paraná, 2016/2017.

\begin{tabular}{|c|c|c|c|}
\hline Variáveis & $\mathbf{n}$ & $(\%)^{\star}$ & IC $95 \%{ }^{*}$ \\
\hline \multicolumn{4}{|l|}{ Demográficas } \\
\hline \multicolumn{4}{|l|}{ Sexo } \\
\hline Masculino & 638 & 51,0 & 45,$0 ; 57,0$ \\
\hline Feminino & 594 & 49,0 & 43,$0 ; 55,0$ \\
\hline \multicolumn{4}{|l|}{ Faixa etária } \\
\hline 10-13 anos & 568 & 49,8 & 31,$8 ; 67,8$ \\
\hline $14-16$ anos & 532 & 41,1 & 26,$9 ; 56,9$ \\
\hline 17 ou mais & 122 & 9,1 & 4,$9 ; 16,3$ \\
\hline \multicolumn{4}{|l|}{ Turno escolar e trabalho } \\
\hline \multicolumn{4}{|l|}{ Turno escolar } \\
\hline Matutino & 720 & 57,1 & 35,$4 ; 76,4$ \\
\hline Vespertino & 373 & 34,4 & 16,$8 ; 57,6$ \\
\hline Intermediário/ Integral & 115 & 8,5 & 2,$1 ; 28,8$ \\
\hline \multicolumn{4}{|l|}{ Trabalho remunerado } \\
\hline Não & 1029 & 86,8 & 81,$7 ; 90,6$ \\
\hline Sim & 159 & 13,2 & 9,$4 ; 18,3$ \\
\hline \multicolumn{4}{|l|}{$\begin{array}{l}\text { Comportamentos } \\
\text { relacionados à saúde }\end{array}$} \\
\hline \multicolumn{4}{|l|}{$\begin{array}{l}\text { Prática de esporte com } \\
\text { treinador }\end{array}$} \\
\hline Não & 332 & 27,0 & 24,$4 ; 29,7$ \\
\hline Sim & 865 & 73,0 & 70,$3 ; 75,6$ \\
\hline \multicolumn{4}{|l|}{ Experimentação de álcool } \\
\hline Não & 967 & 81,4 & 75,$4 ; 86,3$ \\
\hline Sim & 214 & 18,6 & 13,$7 ; 24,6$ \\
\hline \multicolumn{4}{|l|}{ Experimentação de fumo } \\
\hline Não & 1114 & 94,3 & 91,$8 ; 96,0$ \\
\hline Sim & 74 & 5,7 & 4,$0 ; 8,2$ \\
\hline
\end{tabular}

prevalência de consumo regular do café da manhã entre os adolescentes que: não realizavam prática de esporte com treinador; realizavam a maioria das refeições em outros locais que não o domicílio; não tinham experimentado álcool e; entre aqueles com idade de 14 a 16 anos. Por outro lado, maiores frequências foram observadas entre os adolescentes dos turnos vespertino e intermediário ou integral, comparados àqueles do turno matutino, bem como entre aqueles que referiram dormir oito horas ou mais por noite. Após análise ajustada permaneceram associadas ao menor consumo regular de café da manhã: a faixa etária entre 14 e 16 anos, e realizar a maioria das refeições fora de casa. Em contrapartida, $\mathrm{o}$ consumo regular de café da manhã foi mais frequente entre aqueles que referiram dormir mais de oito horas por noite, realizar quatro ou mais

Tabela 1. Distribuição das características de adolescentes das escolas estaduais de Curitiba, Paraná, 2016/2017.

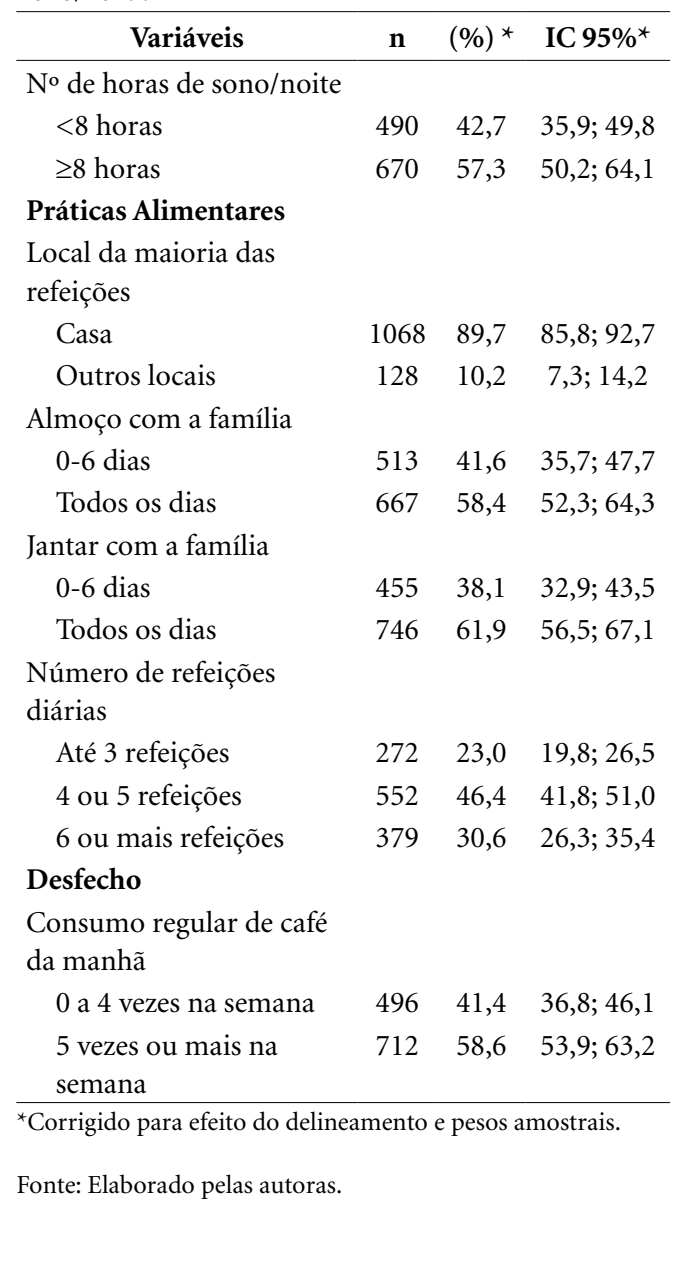


Tabela 2. Associação de variáveis com consumo regular de café da manhã em adolescentes de escolas estaduais, Curitiba, Paraná, 2016/2017.

\begin{tabular}{|c|c|c|c|c|c|}
\hline Variáveis & $\begin{array}{c}\text { Consumo café } \\
\text { da manhã } 5 \text { ou } \\
\text { mais vezes na } \\
\text { semana } n(\%)^{*}\end{array}$ & $\begin{array}{l}\text { RP bruta } \\
\text { (IC 95\%) }\end{array}$ & $\begin{array}{l}\text { Valor } \\
\text { de } \mathbf{p}^{\star \star}\end{array}$ & $\begin{array}{l}\text { RP ajustada } \\
\text { (IC 95\%) }\end{array}$ & $\begin{array}{c}\text { Valor } \\
\text { de } p^{\star \star}\end{array}$ \\
\hline \multicolumn{6}{|l|}{ Demográficas } \\
\hline Sexo & & & 0,120 & & $0,154^{1}$ \\
\hline Masculino & $382(61,4)$ & 1,00 & & 1,00 & \\
\hline Feminino & $330(56,1)$ & $1,00(0,82 ; 1,03)$ & & $1,00(0,82 ; 1,03)$ & \\
\hline Faixa etária & & & 0,037 & & $0,052^{1}$ \\
\hline 10-13 anos & $387(53,2)$ & 1,00 & & 1,00 & \\
\hline $14-16$ anos & $279(51,9)$ & $0,80(0,70 ; 0,92)$ & & $0,81(0,71 ; 0,93)$ & \\
\hline 17 ou mais & $73(60,8)$ & $0,95(0,81 ; 1,10)$ & & $0,94(0,80 ; 1,10)$ & \\
\hline \multicolumn{6}{|l|}{ Turno escolar e trabalho } \\
\hline Turno escolar & & & 0,003 & & $0,001^{2}$ \\
\hline Matutino & $387(53,2)$ & 1 & & 1,00 & \\
\hline Vespertino & $245(64,6)$ & $1,21(1,07 ; 1,37)$ & & $1,22(1,07 ; 1,40)$ & \\
\hline Intermediário/Integral & $80(73,1)$ & $1,37(1,12 ; 1,67)$ & & $1,38(1,13 ; 1,69)$ & \\
\hline Trabalho & & & 0,107 & & \\
\hline Sim & $614(59,5)$ & $0,88(0,75 ; 1,03)$ & & & \\
\hline Não & $84(52,4)$ & 1,00 & & $* * *$ & $* * *$ \\
\hline \multicolumn{6}{|c|}{ Comportamentos relacionados à saúde } \\
\hline Prática de esporte com treinador & & & 0,016 & & $0,012^{3}$ \\
\hline Sim & $211(63,1)$ & 1,00 & & 1,00 & \\
\hline Não & $495(57,3)$ & $0,90(0,84 ; 0,98)$ & & $0,89(0,82 ; 0,97)$ & \\
\hline Experimentação de álcool & & & 0,040 & & $0,113^{3}$ \\
\hline Não & $583(60,8)$ & $0,82(0,69 ; 1,00)$ & & $0,85(0,70 ; 1,03)$ & \\
\hline Sim & $114(50,5)$ & 1,00 & & 1,00 & \\
\hline Experimentação de fumo & & & 0,074 & & \\
\hline Não & $668(59,8)$ & $0,81(0,64 ; 1,02)$ & & $* * *$ & $* * *$ \\
\hline Sim & $37(48,7)$ & 1,00 & & & \\
\hline \multicolumn{6}{|l|}{ No de horas de sono/noite } \\
\hline$<8$ horas & $242(49,8)$ & 1,00 & $<0,001$ & 1,00 & $<0,001^{3}$ \\
\hline$\geq 8$ horas ou mais & $441(65,7)$ & $1,30(1,15 ; 1,45)$ & & $1,32(1,18 ; 1,46)$ & \\
\hline \multicolumn{6}{|l|}{ Práticas Alimentares } \\
\hline Local da maioria das refeições & & & 0,025 & & $0,042^{4}$ \\
\hline Casa & $637(59,9)$ & 1,00 & & 1,00 & \\
\hline Outros locais & $66(49,6)$ & $0,82(0,70 ; 0,97)$ & & $0,82(0,69 ; 1,00)$ & \\
\hline Almoço com a família & & & 0,001 & & $0,009^{4}$ \\
\hline 0 a 6 dias & $263(59,9)$ & 1,00 & & 1,00 & \\
\hline Todos os dias & $430(64,7)$ & $1,26(1,10 ; 1,44)$ & & $1,19(1,05 ; 1,36)$ & \\
\hline Jantar com a família & & & $<0,001$ & & $0,023^{4}$ \\
\hline 0 a 6 dias & $227(49,5)$ & 1,00 & & 1,00 & \\
\hline Todos os dias & $478(64,6)$ & $1,30(1,16 ; 1,45)$ & & $1,15(1,02 ; 1,31)$ & \\
\hline Número de Refeições Diárias & & & $<0,001$ & & $<0,001^{4}$ \\
\hline Até 3 refeições & $113(42,4)$ & 1,00 & & 1,00 & \\
\hline 4 ou 5 refeições & $343(62,7)$ & $1,47(1,23 ; 1,77)$ & & $1,47(1,23 ; 176)$ & \\
\hline 6 ou mais refeições & $248(64,7)$ & $1,52(1,27 ; 1,81)$ & & $1,50(1,25 ; 1,79)$ & \\
\hline
\end{tabular}

Consumo regular $=5$ ou mais vezes na semana; ${ }^{*}$ Teste do Qui-quadrado de Pearson corrigido para efeito do delineamento; ${ }^{* *}$ Teste de Wald; Regressão de Poisson corrigida para efeito do delineamento e pesos amostrais; 1: Ajustado para variáveis demográficas; 2 : Ajustado para variáveis demográficas e turno escolar e trabalho; 3: Ajustado para variáveis demográficas, turno escolar e trabalho e comportamentos relacionados à saúde; 4: Ajustado para variáveis demográficas, turno escolar e trabalho, comportamentos relacionados à saúde e práticas alimentares. ${ }^{* * *}$ valor de $\mathrm{p}>0,25$ na análise ajustada. 
refeições diárias, bem como indicaram almoçar diariamente com a família.

A frequência de consumo regular de café da manhã esteve associada ao consumo de peixes/ mariscos, hortaliças cruas, batata/mandioca/aipim cozidos, legumes cozidos, leite e derivados, bolacha doce, recheios de pão e frutas frescas. Após análise ajustada a prevalência do desfecho permaneceu associada ao maior consumo de leite e derivados e ao menor consumo de macarrão instantâneo e de refrigerantes (Tabela 3).

\section{Discussão}

A prevalência de consumo não regular do café da manhã observada no presente estudo $(41,4 \%)$ foi próxima àquela encontrada para omissão desta refeição no dia anterior, encontrada em estudo com adolescentes de São Paulo $(38,0 \%)^{10}$. Por sua vez, a prevalência de consumo regular do café da manhã $(58,6 \%)$ encontrava-se dentro da faixa de proporção de adolescentes que consumiam o café da manhã diariamente $(45,0 \%$ a $95,0 \%)$, observada nos estudos selecionados na revisão de
Rampersaud et al. ${ }^{3}$, e acima do valor encontrado entre adolescentes brasileiros (48,5\%) no Estudo de Riscos Cardiovasculares em Adolescentes (ERICA $)^{15}$.

A comparação da prevalência de consumo de café da manhã entre pesquisas é limitada devido à grande variedade de definições ${ }^{10}$ utilizadas para caracterizar os consumidores de café da manhã nos estudos, incluindo: consumo diário em casa ou na escola; todos os dias letivos; ingestão no dia da pesquisa; um número mínimo de vezes na semana ou; consumo usual ou habitual, além da própria definição do conceito de café da manhã utilizada pelos adolescentes ao responderem à questão.

Neste estudo, a menor frequência de consumo regular do café da manhã, de acordo com o aumento da idade nos adolescentes, foi semelhante àquela encontrada na revisão de Trancoso et al. ${ }^{9}$, na qual os autores observaram diminuição do consumo com o aumento da idade, na faixa etária dos 4 aos 18 anos. O mesmo ocorreu em estudo com adolescentes alemães, que registrou diminuição significativa da frequência de consumo regular de café da manhã com a idade, nas

Tabela 3. Associação do consumo de alimentos com o consumo regular de café da manhã em adolescentes de escolas estaduais. Curitiba (PR), 2017/2018.

\begin{tabular}{|c|c|c|c|c|}
\hline Variáveis & $\begin{array}{c}\text { RP bruta } \\
\text { (IC95\%) }\end{array}$ & $\begin{array}{l}\text { Valor } \\
\text { de } p^{\star}\end{array}$ & $\begin{array}{l}\text { RP ajustada } \\
* *(\text { IC 95\%) }\end{array}$ & $\begin{array}{l}\text { Valor } \\
\text { de }^{\star \star \star \star}\end{array}$ \\
\hline Macarrão instantâneo & $0,96(0,93 ; 1,00)$ & 0,051 & $0,93(0,89 ; 0,98)$ & 0,009 \\
\hline Carnes & $1,00(0,97 ; 1,04)$ & 0,640 & $* * *$ & $* * *$ \\
\hline Peixes/mariscos & $1,03(1,00 ; 1,06)$ & 0,015 & $1,01(0,91 ; 1,04)$ & 0,177 \\
\hline Batata frita/mandioca frita/polenta frita/banana frita & $1,00(0,80 ; 0,98)$ & 0,808 & $* * *$ & $* * *$ \\
\hline Hortaliças cruas & $1,03(1,01 ; 1,05)$ & 0,004 & $* * *$ & $* * *$ \\
\hline Batata cozida/polenta/aipim cozido & $1,02(1,00 ; 1,05)$ & 0,040 & $1,01(0,98 ; 1,03)$ & 0,240 \\
\hline Legumes cozidos & $1,03(1,00 ; 1,06)$ & 0,014 & $* * *$ & $* * *$ \\
\hline Recheios de pão & $1,03(1,01 ; 1,06)$ & 0,007 & $1,02(0,98 ; 1,05)$ & 0,174 \\
\hline Lanches prontos & $0,98(0,95 ; 1,01)$ & 0,238 & $* * *$ & $* * *$ \\
\hline Leite e derivados & $1,06(1,03 ; 1,09)$ & $<0,001$ & $1,06(1,03 ; 1,10)$ & $<0,001$ \\
\hline Frutas frescas & $1,03(1,00 ; 1,06)$ & 0,012 & $* * *$ & $* * *$ \\
\hline Suco de frutas natural/polpa & $1,01(0,98 ; 1,03)$ & 0,394 & $* * *$ & $* * *$ \\
\hline Refrigerante & $0,97(0,94 ; 0,99)$ & 0,042 & $0,97(0,94 ; 0,99)$ & 0,039 \\
\hline Salgados fritos & $0,98(0,95 ; 1,01)$ & 0,201 & $* * *$ & $* * *$ \\
\hline Doces/balas/sobremesas & $0,99(0,96 ; 1,01)$ & 0,498 & $* * *$ & $* * *$ \\
\hline Embutidos & $0,99(0,97 ; 1,01)$ & 0,590 & $* * *$ & $* * *$ \\
\hline Biscoito doce & $1,01(0,98 ; 1,03)$ & 0,448 & $* * *$ & $* * *$ \\
\hline Feijão/ervilha/lentilha & $1,01(0,99 ; 1,03)$ & 0,283 & $* * *$ & $* * *$ \\
\hline Biscoito salgado & $1,01(0,99 ; 1,03)$ & 0,122 & $* * *$ & $* * *$ \\
\hline Salgadinho ou batata chips de pacote & $0,99(0,96 ; 1,02)$ & 0,633 & $* * *$ & $* * *$ \\
\hline
\end{tabular}

Consumo regular $=5$ ou mais vezes na semana; ${ }^{*}$ Teste de Wald; Regressão de Poisson corrigida para efeito do delineamento e pesos amostrais; ${ }^{*}$ Ajustado para variáveis demográficas, turno escolar e trabalho, comportamentos relacionados à saúde, práticas alimentares e consumo de alimentos; ${ }^{* * *}$ Variáveis com valor de $\mathrm{p}>0,25$ na análise bruta ou ajustada não incluídas no modelo final. 
faixas etárias de 6 a 12 e 13 a 18 anos $^{16}$. Embora alguns estudos tenham encontrado maior omissão do café da manhã entre meninas ${ }^{7,15}$, não foi observada diferença significativa no consumo regular da refeição de acordo com o sexo entre os adolescentes que participaram da presente pesquisa. As explicações para a maior omissão do café da manhã no sexo feminino encontradas em outros estudos estão relacionadas às tentativas de reduzir peso corporal, ainda que esta não seja uma estratégia considerada efetiva pela literatura científica $^{17}$.

Os adolescentes que referiram realizar a maioria das refeições em casa, ou alguma das principais refeições do dia com a família (almoço ou jantar), apresentaram maior frequência de consumo regular do café da manhã. Além do comer, estas ocasiões costumam envolver, a compra dos alimentos, a preparação, o conversar, e proveem oportunidade para que os pais apresentem comportamentos alimentares saudáveis que servirão de modelo para os filhos ${ }^{18,19}$. Por outro lado, omitir o café da manhã apresenta relação com aumento do consumo de lanches calóricos (ricos em carboidratos e gorduras), e o consumo não regular desta refeição torna as crianças menos propensas a almoçar e/ou jantar ${ }^{15}$.

Também foi observado que os adolescentes que apresentaram consumo regular de café da manhã relataram maior consumo de leite e derivados e de recheios de pão. No Brasil, a composição dessa refeição pode ser simplificada pelo consumo de café com leite e pão com manteiga ou margarina ${ }^{10}$ e assemelha-se muito com os alimentos mais consumidos no café da manhã de adultos acima de 20 anos $^{20}$. Por outro lado, o consumo regular do desjejum foi inversamente associado à ingestão de refrigerantes, o que reforça a realização do café da manhã como marcador de hábitos alimentares saudáveis. Ao interpretar os resultados referentes ao consumo de alimentos e do café da manhã, encontrados no presente estudo, não foi possível estabelecer se os alimentos foram consumidos no café da manhã ou em outros horários do dia, pois foi aplicado questionário de frequência de consumo de alimentos, e não recordatório alimentar de 24 horas.

O consumo do café da manhã pode melhorar a sensação de saciedade do comensal, e assim reduzir a quantidade calórica total ingerida durante o dia; em especial, pode limitar o consumo de lanches calóricos, por crianças e adolescentes diariamente ${ }^{9}$. Parece também auxiliar no controle de peso e, se comparada aos lanches, a refeição matinal proporciona uma maior ingestão de vi- taminas e minerais e menor ingestão de gorduras e colesterol'.

A ausência dessa refeição pode, por sua vez, inviabilizar a elevação da glicemia, necessária às atividades matinais, e favorecer uma possível deficiência de cálcio, uma vez que nessa refeição geralmente se concentra o maior consumo diário de leites e derivados, que são fontes desse mine$\mathrm{ral}^{3}$. Do mesmo modo, sua substituição por lanches durante o dia não é vista como uma prática saudável. Alguns estudos mostram que essa substituição, principalmente entre crianças e adolescentes comparados com indivíduos que ingeriam habitualmente o café da manhã teve como consequência o aumento do consumo energético total de carboidratos, especialmente os carboidratos refinados e as gorduras ${ }^{21,22}$.

Além das questões alimentares, outros comportamentos considerados de risco à saúde também foram significativamente associados ao perfil de adolescentes e adultos que não consumiam café da manhã em outros estudos: fumar; não praticar exercícios físicos com frequência; fazer uso frequente de álcool ${ }^{7} \mathrm{e}$; dormir menor número de horas por noite ${ }^{23,24}$. Uma associação inversa entre consumo de café da manhã e tabagismo e comportamento de beber álcool foi documentada nos estudos de Kapantais ${ }^{25}$, Keski -Rahkonen ${ }^{4}$ e Timlin ${ }^{26}$. Esses resultados reforçam aqueles encontrados no presente estudo, no qual observou-se que adolescentes que referiram ter experimentado fumo e bebidas alcoólicas referiram consumo menos frequente do desjejum, enquanto aqueles que realizavam prática de esporte com treinador relataram maior consumo. Parte da associação com uso de fumo e bebidas alcoólicas pode ser explicada pela idade dos adolescentes que já experimentaram os produtos, e que realizam com menor frequência o café da manhã, o que foi demonstrado a partir da análise ajustada, pois a associação das variáveis com o desfecho deixou de ser significativa. Entre os adolescentes avaliados, o menor número de horas de sono esteve associado a menor prevalência de consumo regular de café da manhã, resultado similar àquele observado em outros estudos ${ }^{23,24}$. Adolescentes que dormem mais horas por noite podem ter escolhas alimentares melhores durante o café da manhã ${ }^{27}$.

Ao se interpretar os resultados do presente estudo é necessário cautela, devido ao seu delineamento transversal. Desta forma, não é possível estabelecer relação de causa e efeito entre o consumo de café da manhã e as associações observadas. Ainda assim, por exemplo, a associa- 
ção mais forte com alimentos tradicionalmente consumidos neste horário, como leite e derivados e recheios de pão, indicam melhores escolhas alimentares e menor consumo calórico durante o dia em outras refeições. Os resultados encontrados reforçam o café da manhã como marcador de alimentação e de outros hábitos saudáveis em adolescentes.

Por fim, os achados do presente estudo reforçam as recomendações de Rampersaud et al. ${ }^{3} \mathrm{em}$ seu estudo de revisão sobre o consumo de café da manhã para crianças e adolescentes, destacandose a necessidade de consumir diariamente um café da manhã saudável com variedade entre os grupos alimentares, por exemplo, grãos integrais, frutas e produtos lácteos, priorizando o consumo de alimentos nutritivos, ricos em fibras, fonte de cálcio e pobres em carboidratos refinados. Ainda, destaca-se que o incentivo dessa refeição para crianças e adolescentes deve ser contínuo, tanto por parte dos pais como por parte das escolas e programas de incentivo ao consumo de café da manhã.

\section{Conclusões/considerações finais}

Os resultados do presente estudo indicam que o consumo regular do desjejum foi maior para aqueles que: estudavam nos turnos vespertino e integral; realizavam quatro refeições ou mais/ dias, sendo a maioria das refeições com a família, especialmente o jantar todos os dias; referiram não ter experimentado bebidas alcoólicas; realizavam prática de esporte com treinador e; totalizavam mais de oito horas de sono diária.

Por fim, destaca-se a importância de políticas de educação e promoção de comportamentos alimentares saudáveis, incluindo o incentivo para realização do desjejum, já que esse comportamento está associado a um maior consumo de alimentos fonte de nutrientes importantes. Atenção especial deve ser dada aos adolescentes mais velhos que, com maior frequência, omitem essa refeição.

\section{Colaboradores}

Todas as autoras deste artigo participaram diretamente no planejamento, execução e análises deste estudo. Todas as autoras deste artigo leram e aprovaram a versão final. 


\section{Referências}

1. Brasil. Ministério da Saúde (MS). Guia Alimentar para a população brasileira. $2^{\mathrm{a}}$ ed. Brasília: MS; 2014.

2. Kral TV, Whiteford LM, Heo M, Faith MS. Effects of eating breakfast compared with skipping breakfast on ratings of appetite and intake at subsequent meals in 8- to 10-y-old children. Am J Clin Nutri 2011; 93(2):284-291.

3. Rampersaud GC, Pereira MA, Girard BL, Adams J, Metzl J. Breakfast habits, nutritional status, body weight, and academic performance in children and adolescents. J Am Diet Assoc 2005;105(5):743-760.

4. Keski-Rahkonen KJ, Rissanen A, Virkkunen M, Rose RJ. Breakfast skipping and health compromising behaviors in adolescents and adults. Eur J Clin Nutr 2003; 57(7):842-853.

5. Uzhova I, Fuster V, Fernandez O, Ordovás JM, et al. The importance of breakfast in atherosclerosis disease: Insights from the PESA Study. J Am College Cardio$\log y$ 2017; 70(15):1833-1842.

6. Maki KC, Philips-Eakley A.K, Smith KN. The effects of breakfast consumption and composition on metabolic wellness with a focus on carbohydrate metabolism. Adv Nutr 2016; 7(3):613-621.

7. Uemura M, Yatsuya H, Hilawe EH, Li Y, Wang C, Chiang C, Otsuka R, Toyoshima H,Tamakoshi K, Ayoma A. Breakfast Skipping is Positively Associated With Incidence of Type 2 Diabetes Mellitus: Evidence From the Aichi Workers Cohort Study. J Epidemiol 2015; 25(5):351-358

8. Vásquez A, Busto Zapico B, Errasti Pérez JM, Pena Suárez P. Skipping breakfast, sedentarism and overweight in children. Psychol Health Med 2016; 21(7):819826.

9. Trancoso SC, Cavalli SB, Proença RPC. Café da manhã: caracterização, consumo e importância para a saúde. Rev Nutr 2010; 23(5):859-869.

10. Fisberg RM, Marchioni DML, Gorgulho BM, Teixeira JA, Verly E. Prevalência de omissão do café da manhã e seus fatores associados em adolescentes de São Paulo: estudo ISA-Capital. Nutrire 2015; 40(1):10-21.

11. Instituto Brasileiro de Geografia e Estatística (IBGE). Cidades. [acessado 2015 mar 5]. Disponível em: http://www.cidades.ibge.gov.br/xtras/temas.php?lan$\mathrm{g}=\& \operatorname{codmun}=41069 \mathrm{dtema}=118 \&$ search $=$ parana $\mid \mathrm{cu}-$ ritiba|\%C3\%8Dndice-deDesenvolvimentohumano -municipalidhm

12. Secretaria da Educação do Paraná (SEP). Superintendência de desenvolvimento educacional. Resultados do censo escolar - 2014. Série Histórica da Matrícula de Educação Básica no Paraná - 2004. [acessado 2015 mar 5]; 2014. Disponível em: http://www.educacao. pr.gov.br/arquivos/File/Censo/serie_historica2014. pdf

13. Clark L, Dania SL, Jacques DHO. Early, Middle, and Late Adolescents' Perceptions of Their Relationships with Significant Others. J Adolesc Res 1991; 6(3):296315 .

14. Molina MCB, Lopéz PM, Faria CP, Cade NV, Zandonade E. Preditores socioeconômicos da qualidade da alimentação de crianças. Rev Saude Publica 2010; 44(5):785-792.
15. Barufaldi LA, Abreu GZ, Oliveira JS, Santos DF, Fujimori E, Vasconcelos SML, Guedes FA, Tavares BM. ERICA: prevalência de comportamentos alimentares saudáveis em adolescentes brasileiros. Rev Saude Publica 2016; 50(Supl. 1, 6s):327-345.

16. Ute A, Wicher M, Kersting M. Breakfast trends in children and adolescents: frequency and quality. Public Health Nutri 2010; 13(11):1795-1802.

17. Araki EL, Philippi ST, Martinez MF, Estima CCP, Leal GVS, Alvarenga MS. Padrão de refeições realizadas por adolescentes que frequentam escolas técnicas de São Paulo. Rev Paul Pediatr 2011; 29(2).

18. Woodruff SJ, Hanning RM. A review of family meal influence on adolescents' dietary intake. Can J Diet Pract Res 2008; 69(1):14-22.

19. Mattos LL, Martins IS. Consumo de fibras alimentares em população adulta. Rev Saude Publica 2000; 34(1):50-55

20. Gauche H, Calvo MCM, Assis MAA. Ritmos circadianos de consumo alimentar nos lanches e refeições de adultos: aplicação do semanário alimentar. Nutr 2006;19(2):177-185.

21. Rivas PR, Figuero CR, Lanza, TA, Lamuño DG, Fuentes MG. Grupo Avena. Desayuno y almuerzo de los adolescentes escolarizados de Santander. Nutr Hosp 2005; 20(3):217-222

22. Nicklas TA, Morales M, Linares A, Yang SJ, Baranowski T, De Moor C, Berenson G. Children's meal patterns have changed over a 21-year period: the Bogalusa heart study. J Am Diet Assoc 2004; 104(5):753-61.

23. Gong QH, Li H, Zhang T, Cui J, Xu Gs. et al. Associations between sleep duration and physical activity and dietary behaviors in Chinese adolescents: results from the Youth Behavioral Risk Factor Surveys of 2015. Sleep Medicine 2017; 37:168-173.

24. Ogilvie RP, Redline S, Bertoni AG. Sleep indices and eating behaviors in young adults: findings from Project EAT. Public Health Nutri 2017; 21(04):689-701.

25. Kapantais E, Chala E, Kaklamanou D, Lanaras L, Kaklamanou M, Tzotzas T. Breakfast skipping and its relation to BMI and health-compromising behaviours among Greek adolescents. Public Health Nutr 2011; 14(1):101-108.

26. Timlin MT, Pereira MA, Story M, Neumark-Sztainer D. Breakfast eating and weight change in a 5-year prospective analysis of adolescents: Project EAT (Eating among Teens) Pediatrics. 2008; 121:638-645.

27. Asarnow LD, McGlinchey E, Harvey AG. The Impact of Sleep Improvement on Food Choices in Adolescents With Late Bedtimes. J Adolesc Health 2017; 60(5):570-576.

Artigo apresentado em 29/08/2018

Aprovado em 29/07/2019

Versão final apresentada em 31/07/2019

Editores-chefes: Romeu Gomes, Antônio Augusto Moura da Silva 
